\title{
Falls efficacy, postural balance, and risk for falls in older adults with falls-related emergency department visits: prospective cohort study
}

Yong-Hao Pua ${ }^{1 *}$, Peck-Hoon Ong ${ }^{1}$, Ross Allan Clark², David B. Matcher ${ }^{3}$ and Edwin Choon-Wyn Lim

\begin{abstract}
Background: Risk for falls in older adults has been associated with falls efficacy (self-perceived confidence in performing daily physical activities) and postural balance, but available evidence is limited and mixed. We examined the interaction between falls efficacy and postural balance and its association with future falls. We also investigated the association between falls efficacy and gait decline.

Methods: Falls efficacy, measured by the Modified Falls Efficacy Scale (MFES), and standing postural balance, measured using computerized posturography on a balance board, were obtained from 247 older adults with a falls-related emergency department visit. Six-month prospective fall rate and habitual gait speed at 6 months post baseline assessment were also measured.

Results: In multivariable proportional odds analyses adjusted for potential confounders, falls efficacy modified the association between postural balance and fall risk (interaction $P=0.014$ ): increasing falls efficacy accentuated the increased fall risk related to poor postural balance. Low baseline falls efficacy was strongly predictive of worse gait speed $(0.11 \mathrm{~m} / \mathrm{s}$ [0.06 to 0.16 ] slower gait speed per IQR decrease in MFES; $P<0.001)$.

Conclusion: Older adults with high falls efficacy but poor postural balance were at greater risk for falls than those with low falls efficacy; however, low baseline falls efficacy was strongly associated with worse gait function at follow-up. Further research into these subgroups of older adults is warranted.
\end{abstract}

Trial registration: ClinicalTrials.gov identifier: NCT01713543.

Keywords: Balance, Falls efficacy, Falls

\section{Background}

Falls are a common reason for older adults attending the emergency department (ED), and older adults with a falls-related ED visit have increased risk for subsequent falls, hospital readmission, functional decline, and mortality [1-5]. Accordingly, identifying the risk factors for falls in this clinical population is crucial [6].

Although low falls efficacy, defined as low selfperceived confidence in engaging in activities of daily living without falling, and impaired postural balance are

\footnotetext{
* Correspondence: puayonghao@gmail.com

'Department of Physiotherapy, Singapore General Hospital, Outram Road,

Singapore 169608, Singapore

Full list of author information is available at the end of the article
}

putative risk factors for falls in community-dwelling older adults, the available data are limited and inconsistent [7-9]. We believe, however, that the mixed results of previous studies could reflect the complex interplay between falls efficacy, postural balance, and fall risk. Specifically, older adults with low falls efficacy may restrict their daily activities [9] - a strategy that may lower fall risk at least in the short term and diminish the association between postural balance and fall risk. However, this activity avoidance could potentially lead to functional decline over time. Conversely, while high falls efficacy is generally thought to be protective against falls, fall risk may be heightened among older adults with high falls efficacy but poor postural balance - a subgroup of 
"over-confident" older adults that has been largely neglected in previous falls studies [10-12].

Thus, our study aimed to examine the associations of falls efficacy, postural balance, and their interaction with fall risk (defined as the number of incident falls over a 6-month period) in a sample of older adults with fallsrelated ED visits. To further clarify the clinical significance of falls efficacy, we examined its association with future gait limitations.

\section{Methods}

The current investigation was performed as a substudy of the Steps to Avoid Falls in Elderly (SAFE) trial (ClinicalTrials.gov NCT01713543) [13], a multicenter randomized trial that compared a home-based, customised programme versus the provision of an education booklet. Between December 2012 and June 2014, study personnel identified community-dwelling older adults ( $\geq 65$ years) presenting to and discharged from the ED for a fall or fall-related injury. Exclusion criteria were an inability to follow the three-step command test, non-ambulatory status before the ED visit, and total blindness Trained research personnel travelled to participants' home and assessed participants using a battery of self-report and functional measures at 3 and 9 months following hospital discharge. Due to limited personnel and funding, convenience sampling was used and the present study included 247 participants with available postural balance data at 3 months following hospital discharge (hereafter known as the baseline measure). Included participants were similar to those who were excluded because of missing postural balance data (data not shown). The study was approved by the institutional review boards of all participating sites, and all participants provided written informed consent.

\section{Falls efficacy and postural balance}

We examined two primary independent risk factors: baseline falls efficacy and postural balance. Falls efficacy was measured with the 14-item Modified Falls Efficacy Scale (MFES) questionnaire [14]. Each item was scored on a 10-point Likert scale and the score of all answered items were summed and adjusted to a 0 to 140 scale, with higher scores indicating greater falls efficacy. The MFES was selected over the other scales because its items included daily outdoor activities on the use of public transport and road crossing. Standing postural balance was measured using computerized posturography on a Nintendo Wii Balance Board $[15,16]$, which has been shown to have concurrent validity with laboratory force plates [16]. Participants stood quietly on the balance board for $30 \mathrm{~s}$ and centre-of-pressure velocity along the anteroposterior (velocity-AP) and medio-lateral (velocity-ML) planes was recorded. Conceptually, centre-of-pressure velocity represents the amount of activity required to maintain stability during quiet standing; hence, greater velocity indicates poorer balance [17]. All participants performed 3 trials and the mean was taken.

\section{Covariates}

To adjust for potential confounding (by risk factors for falls), we used subject-matter expertise and the literature [18-22] to a priori identify 6 covariates - namely, age [19], sex [18], fall history (yes/no) within the past 9 months (but excluding the most recent fall episode) [22], number of comorbidities [20], baseline Short Physical Performance Battery (SPPB) [21], and treatment group assignment. For the comorbidity measure, we used patient reports to aggregate the presence of 18 comorbid diseases such as hypertension, diabetes, congestive heart failure, coronary artery disease, stroke, Parkinson's disease, and lung diseases. The SPPB $[21,23]$ is a measure of lower-extremity performance and comprises a tandem balance test, a timed 4-m gait speed test, and a timed chair stand test. Each test is scored from 0 to 4 , and the total score ranges from 0 (worst performance) to 12 (best performance).

\section{Outcomes}

The main outcome of the study was incident falls, defined as "an event which results in a person coming to rest inadvertently on the ground or floor of other lower level" [24]. Over a 6-month period, participants tracked monthly fall incidence on fall calendars [25, 26] and were contacted through monthly telephone calls [27]. In addition, we analyzed habitual gait speed, derived from the SPPB, at 6 months post baseline.

\section{Statistical analysis}

Continuous variables were presented as means with standard deviation (SD)s and medians with interquartile range (IQR)s whilst categorical variables were presented as frequencies with percentages. The distributions of demographic and clinical factors in patients with and without a fall in the follow-up period were compared using the Wilcoxon rank-sum test or $x^{2}$ test, when appropriate.

The independent association of falls efficacy and postural balance with fall risk was assessed using a proportional odds regression model, with the number of falls as the dependent variable and MFES, center of pressure (CoP) AP-velocity, and CoP ML-velocity measures were each used as an independent variable. We adjusted all models for age, sex, fall history, number of comorbidities, baseline SPPB, and treatment group assignment. Proportional odds regression was chosen given its ability to handle zero-inflated falls data in this study [28, 29]. To evaluate the interaction between MFES and postural balance on the 
Table 1 Demographics and clinical characteristics

\begin{tabular}{|c|c|c|c|c|}
\hline & Non-fallers $(n=190)$ & Fallers $(n=57)$ & Overall $(n=247)$ & $P$-value \\
\hline Age (years) & $71.077 .083 .0(77.3 \pm 7.3)$ & $74.078 .083 .0(78.2 \pm 6.2)$ & $73.077 .083 .0(77.5 \pm 7.1)$ & $0.38^{1}$ \\
\hline Women & $75 \%(143)$ & $74 \%(42)$ & $75 \%(185)$ & $0.81^{2}$ \\
\hline Stroke or Parkinson's disease & $6 \%(12)$ & $9 \%(5)$ & $7 \%(17)$ & $0.53^{2}$ \\
\hline Number of comorbidities & $1.02 .04 .0(2.6 \pm 1.6)$ & $2.03 .04 .0(2.8 \pm 1.6)$ & $1.02 .04 .0(2.6 \pm 1.6)$ & $0.32^{1}$ \\
\hline History of previous falls & $43 \%(81)$ & $60 \%(34)$ & $47 \%(115)$ & $0.024^{2}$ \\
\hline Baseline SPPB ${ }^{a}$ & $4.07 .09 .0(6.8 \pm 3.1)$ & $3.05 .08 .0(5.7 \pm 3.0)$ & $4.07 .09 .0(6.5 \pm 3.1)$ & $0.025^{1}$ \\
\hline Baseline MFES ${ }^{b}$ & $78,115,128(100 \pm 34)$ & $66,104,128(95 \pm 33)$ & $76, \mathbf{1 1 2}, 128(99 \pm 34)$ & $0.35^{1}$ \\
\hline Baseline CoPc Velocity-AP $(\mathrm{cm} / \mathrm{s})$ & $0.730 .911 .24(1.11 \pm 0.66)$ & $0.751 .031 .44(1.26 \pm 0.81)$ & $0.730 .951 .29(1.15 \pm 0.70)$ & $0.23^{1}$ \\
\hline Baseline CoP ${ }^{c}$ Velocity-ML $(\mathrm{cm} / \mathrm{s})$ & $0.45 \mathbf{0 . 5 4} 0.69(0.64 \pm 0.37)$ & $0.49 \mathbf{0 . 5 8} 0.85(0.70 \pm 0.31)$ & $0.45 \mathbf{0 . 5 4} 0.74(0.65 \pm 0.35)$ & $0.066^{1}$ \\
\hline Gait speed at 6 months $(\mathrm{m} / \mathrm{s})$ & $0.420 .610 .81(0.59 \pm 0.26)$ & $0.31 \mathbf{0 . 5 0} 0.64(0.50 \pm 0.25)$ & $0.38 \mathbf{0 . 5 6} 0.77(0.57 \pm 0.26)$ & $0.008^{1}$ \\
\hline
\end{tabular}

Continuous variables are summarized as 25 th 50 th 75 th percentiles and mean \pm SD

Median value is captured in boldface

${ }^{1}$ Wilcoxon rank sum test; ${ }^{2}$ Pearson's $x^{2}$ test

${ }^{a}$ SPPB - short Physical Performance Battery; ranges from 0 to 12, with higher scores indicating greater physical functioning

${ }^{\mathrm{b}}$ MFES - modified Falls Efficacy Scale; ranges from 0 to 140, with higher scores indicating greater falls efficacy

${ }^{\mathrm{C}} \mathrm{CoP}$ - centre of pressure

${ }^{\mathrm{d}} \mathrm{AP}$ - anteroposterior

${ }^{\mathrm{e}} \mathrm{ML}$ - mediolateral

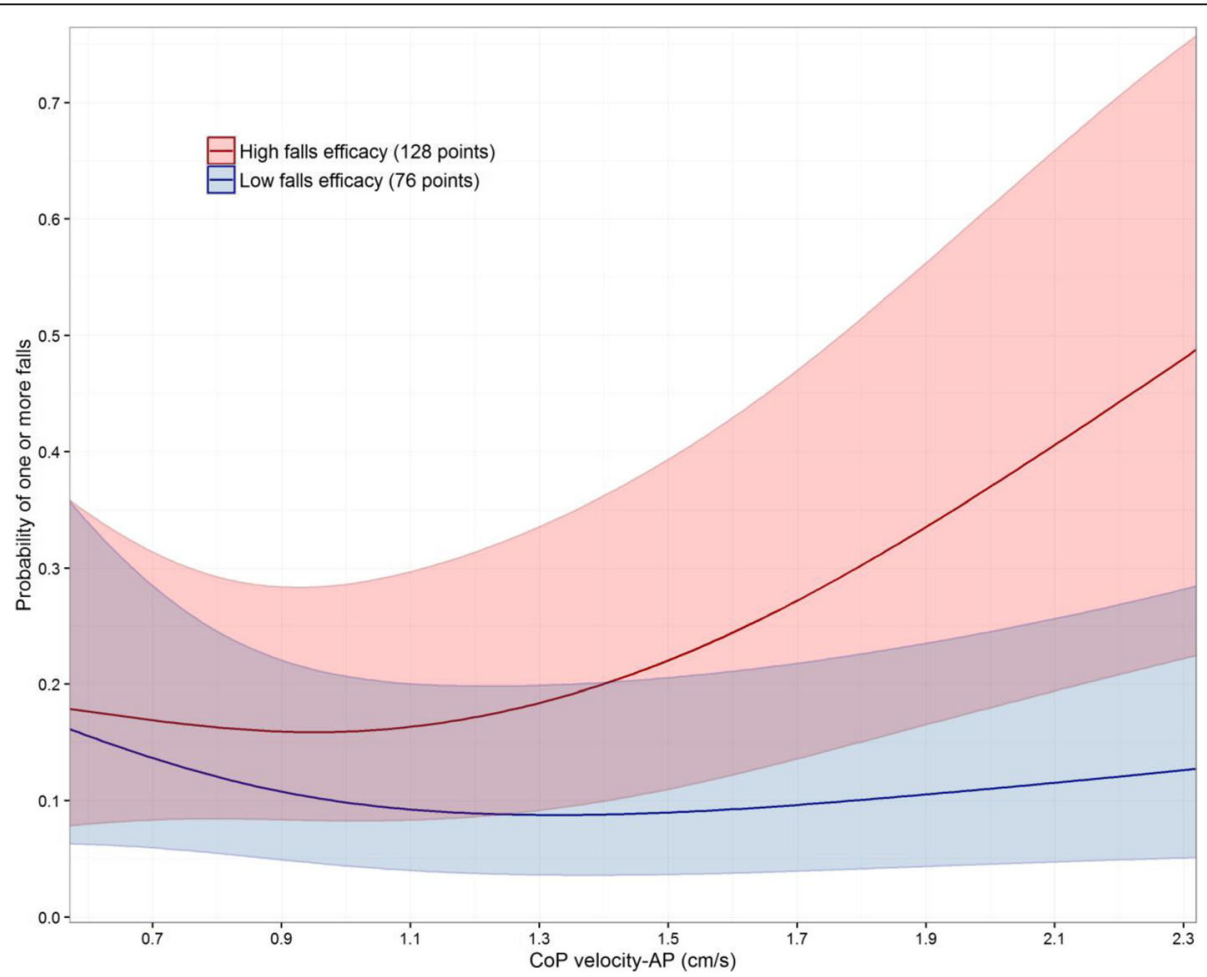

Fig. 1 Interaction plot of Modified Falls Efficacy Scale (MFES) and centre-of-pressure (CoP) velocity-AP and probability of falling at least once in the follow-up period. Low and high falls efficacy represent the 25th and 75th percentile values of the MFES, respectively. Predicted fall risk was computed from a proportional odds model which included the interaction between MFES and CoP velocity-AP, adjusted for age, sex, number of comorbidities, fall history, baseline SPPB, and treatment group assignment ( $P=0.014$ for interaction). Shaded regions represent $95 \%$ Cls for the natural spline-smoothed estimates 
fall outcome, we tested for MFES $\times$ AP-velocity and MFES $\times$ ML-velocity interactions in separate models.

The independent association of falls efficacy with gait speed was assessed using a multivariable linear regression model, with gait speed as the dependent variable and MFES as the independent variable. The two postural balance measures served as covariates and to reduce collinearity, we used principal component analysis to combine them into one variable. Other covariates in the model were age, sex, fall history, number of comorbidities, baseline gait speed, and treatment group assignment.

For all analyses, to avoid assuming linearity, we modeled all continuous predictors as restricted cubic splines [28] unless there was insufficient evidence against the linearity assumption $(P>0.20)$. To minimize selection bias from missing predictor and outcome values, we performed predictive-mean-matching multiple imputation with 50 replications $[28,30]$. In sensitivity analyses, we checked for interactions of treatment group assignment with falls efficacy and postural balance and found no statistically significant effect modification. We assessed the appropriateness of all models using residual plots, and we used $R$ software, version 3.2.3 (http://www.r-project.org), for all analyses and graphing.

\section{Results}

Table 1 shows the demographic and clinical characteristics of the sample. Of the 247 participants, 57 (24\%) participants fell at least once, of whom 21 had two or more falls in the 6-month follow-up. Participants who fell at least once were more likely to report having a previous fall, have lower baseline SPPB scores, and walk more slowly at 6 months.

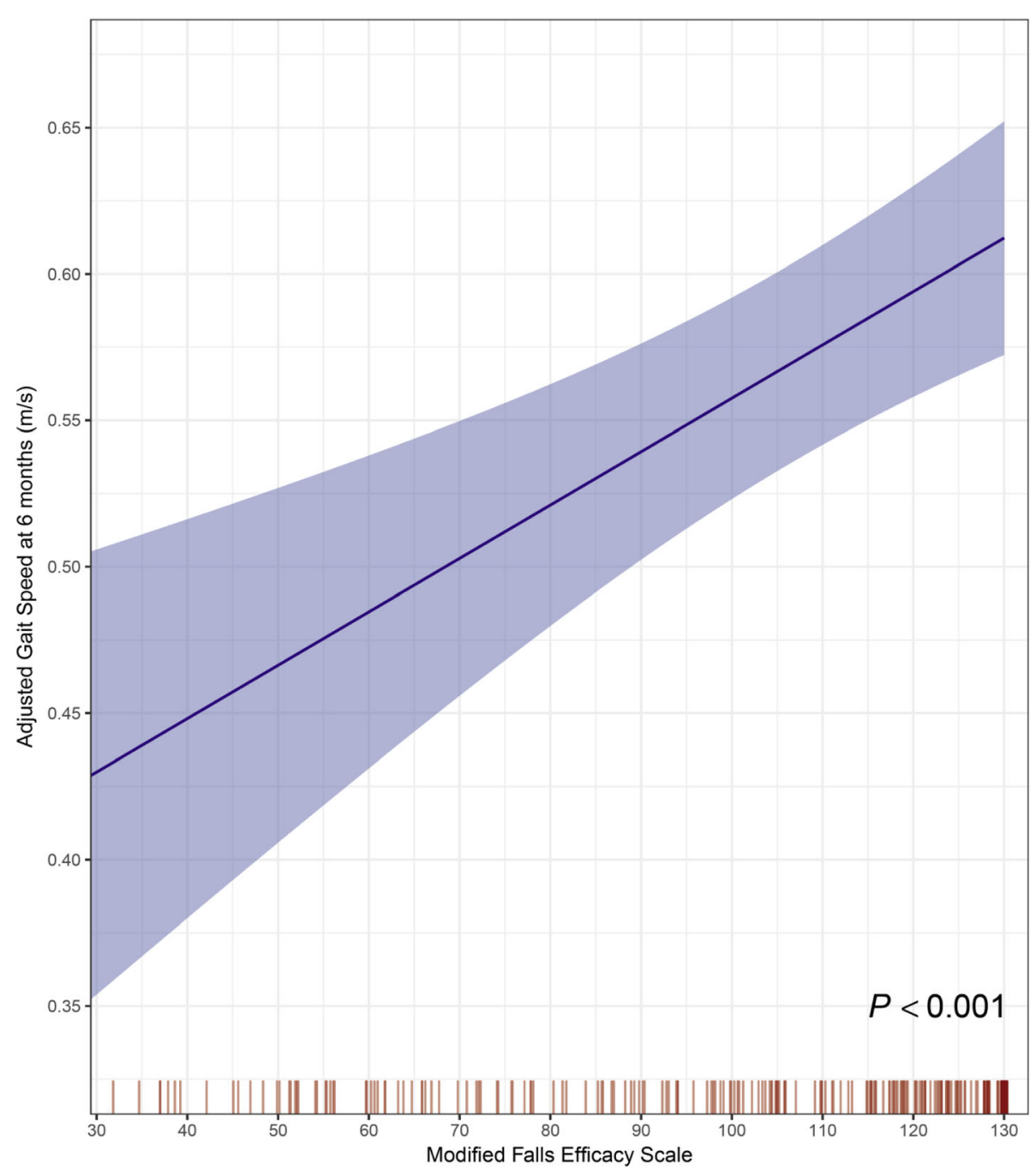

Fig. 2 Lower scores on the Modified Falls Efficacy Scale (MFES) were independently associated with slower (worse) gait speed at 6 months post-baseline assessment, after adjustment for age, sex, number of comorbidities, fall history, baseline gait speed, treatment group assignment, and postural balance. Shaded regions represent $95 \% \mathrm{CI}$ for the point estimates. Rug plots show the observed baseline MFES values 
When adjusted for covariates, there was no significant association between fall risk and MFES (interquartile range-odds ratio [IQR-OR], 1.58 [CI, 0.79 to 3.12], $P=$ 0.19 ), velocity-ML (IQR-OR, 1.00 [CI, 0.77 to 1.29 ]; $P=$ 0.98 ) or velocity-AP measures (IQR-OR, 1.05 [CI, 0.82 to 1.36 ]; $P=0.69)$. The MFES $\times$ velocity-ML interaction was of borderline significance $(P=0.076)$ while MFES interacted significantly with velocity-AP measure to influence fall risk $(P=0.014)$. Specifically, when MFES level was high, greater CoP velocity-AP was closely associated with greater fall risk; however, this association weakened with decreasing MFES levels (Fig. 1).

We then tested whether baseline falls efficacy was associated with gait speed at 6 months post baseline. After adjustment for covariates, lower MFES was an independent risk factor for future gait limitations $(0.11 \mathrm{~m} / \mathrm{s}$ [0.06 to 0.16] slower gait speed per IQR decrease in MFES; $P<0.001$ ) (Fig. 2).

\section{Discussion}

In this prospective study of 247 older adults, baseline falls efficacy interacted with postural balance to influence fall risk. These findings could shed light on the limited and mixed evidence regarding the association of falls efficacy and postural balance with fall risk: if low falls efficacy blunted the association between postural balance and fall risk, the "averaged effects" of falls efficacy and postural balance on fall risk could be biased toward the null.

Reviewing the literature, although falls efficacy has been reported to be significantly reduced in fallers compared to non-fallers [22, 31-33], it was not significantly lower amongst fallers in our study (Table 1). However, we have found that low baseline falls efficacy was strongly predictive of worse gait function (Fig. 2). To explain this phenomenon, it is possible that older adults with low falls efficacy may restrict their daily activities [9] to reduce their fall risk and are less likely to engage in exercises [34] - making them prone to postural instability and muscular weakness associated with deconditioning [35]., Potentially, this sequela of activity restriction may lead to increased long-term fall risk [35, 36]. Conversely, another interpretation is that a slow gait reflects a cautious gait strategy [37] and recent evidence also suggests that the generally low falls efficacy in Chinese older adults is protective against falls [38]. We believe, however, that this alternative explanation is less likely because our participants who fell in the follow-up period had relatively lower baseline SPPB scores and slower follow-up gait speed (Table 1). Nevertheless, to better clarify the causal pathway(s) of our findings, future work should examine the long-term fall risk in older adults with low falls efficacy.

Our results suggest that fall risk was heightened in older adults with high falls efficacy but poor postural balance. This finding is biologically plausible because "over-confident" older adults are also likely to walk fast (Fig. 2) and less likely to restrict their daily activities, and these factors may interact to increase fall risk $[39,40]$. Although Delbaere and colleagues [34] found that their "under-fearful" older adults had similar, if not lower, fall risk compared with older adults who had an accurate perception of their high fall risk, we should emphasize that all our participants had a fall that led to an ED visit. Hence, we speculate that the combination of poor postural balance and high falls efficacy after a recent ( $\sim 3$ months) traumatic fall may more closely reflect a risk taking behaviour or an inaccurate fall-risk appraisal. Nevertheless, we acknowledge that a deeper investigation of our findings would require additional information on physical activity levels and risk-taking behaviour [12], and future work should explore this.

Our study has limitations other than those described earlier. First, although we studied a clinically relevant group of high fall-risk Asian older adults [6], it is unclear whether our results would persist in (i) a non-Asian sample or (ii) a broader sample that includes healthy older adults with no fall history at baseline. Second, whilst we had details about injurious falls and obtained qualitatively similar results (data not shown), our analyses were not sufficiently powered to draw firm conclusions.

\section{Conclusions}

In summary, we observed that older adults with high falls efficacy but poor postural balance were at greater risk for falls than those with low falls efficacy; however, low falls efficacy was strongly predictive of future gait limitations. Further research into these subgroups of older adults is warranted to uncover ways in which their risk factors may be modified.

\section{Abbreviations}

ED: Emergency department; MFES: Modified Falls Efficacy Scale; SAFE trial: Steps to Avoid Falls in Elderly trial; SPPB: Short Physical Performance Battery; velocity-AP: Velocity along the antero-posterior plane; velocity-ML: velocity along the medio-lateral plane

\section{Acknowledgements \\ The authors acknowledge the support from Bee-Yee Tan, the head of the Department of Physiotherapy, Singapore General Hospital. The authors thank Jia-Ying Ho, Jasper Quek, and the geriatrics team of the Department of Physiotherapy, Singapore General Hospital, Rita Sim, research associate, Health Services and System Research, Duke-NUS, Graduate Medical School, Singapore, and the nursing assessors (Kua Lang, Cecilia Leong, and Cheo-Tee Tan) for their contributions to this study. \\ Funding \\ Singapore Ministry of Health research grant (HSRG/10May002) \& Singapore Physiotherapy Association research grant (RF12-001). The funding bodies were not involved in the study design, data collection, analysis, writing of the manuscript or the decision to submit for publication.}

Availability of data and materials

Data are available from the corresponding author on request. 


\section{Authors' contributions}

All authors have contributed to conception and design of this study. YHP performed the statistical analysis and made the first draft of the manuscript. All authors have participated in the interpretation of data. All authors contributed to the critical revision of the manuscript, read, and approved the final manuscript. All authors gave final approval of the version to be published and agreed to be accountable for all aspects of the work in ensuring that questions related to the accuracy or integrity of any part of the work are appropriately investigated and resolved.

\section{Ethics approval and consent to participate}

This study was approved by the centralised institutional review board (2012/147/A) and registered on 24 October 2012 with the U.S. Clinical Trial Registry (NCT01713543). All participants provided written informed consent.

\section{Competing interests}

The authors declare that they have no competing interests.

\section{Publisher's Note}

Springer Nature remains neutral with regard to jurisdictional claims in published maps and institutional affiliations.

\section{Author details}

'Department of Physiotherapy, Singapore General Hospital, Outram Road, Singapore 169608 , Singapore. ${ }^{2}$ Research Health Institute, University of the Sunshine Coast, Sunshine Coast, Australia. ${ }^{3}$ Health Services \& Systems Research Program, Duke-NUS Graduate Medical School, Singapore, Singapore.

\section{Received: 21 February 2017 Accepted: 7 December 2017}

Published online: 21 December 2017

\section{References}

1. Castro VM, McCoy TH, Cagan A, Rosenfield HR, Murphy SN, Churchill SE, et al. Stratification of risk for hospital admissions for injury related to fall: cohort study. BMJ. 2014;349:95863.

2. Ayoung-Chee P, Mclntyre L, Ebel BE, Mack CD, McCormick W, and Maier RV. Long-term outcomes of ground-level falls in the elderly. J Trauma Acute Care Surg 2014; 76: 498-503; discussion 503.

3. Bloch F, Jegou D, Dhainaut JF, Rigaud AS, Coste J, Lundy JE, et al. Do ED staffs have a role to play in the prevention of repeat falls in elderly patients? Am J Emerg Med. 2009;27:303-7.

4. Carpenter CR, Avidan MS, Wildes T, Stark S, Fowler SA, Lo AX. Predicting geriatric falls following an episode of emergency department care: a systematic review. Acad Emerg Med. 2014;21:1069-82.

5. Denkinger MD, Lukas A, Nikolaus T, Hauer K. Factors associated with fear of falling and associated activity restriction in community-dwelling older adults: a systematic review. Am J Geriatr Psychiatry. 2015;23:72-86.

6. Carpenter CR, Shah MN, Hustey FM, Heard K, Gerson LW, miller DK. High yield research opportunities in geriatric emergency medicine: prehospital care, delirium, adverse drug events, and falls. J Gerontol A Biol Sci Med Sci. 2011;66:775-83

7. Piirtola M, Era P. Force platform measurements as predictors of falls among older people - a review. Gerontology. 2006;52:1-16.

8. Deandrea S, Lucenteforte E, Bravi F, Foschi R, La Vecchia C, Negri E. Risk factors for falls in community-dwelling older people: a systematic review and meta-analysis. Epidemiology. 2010;21:658-68.

9. Hughes CC, Kneebone II, Jones F, Brady BA. Theoretical and empirical review of psychological factors associated with falls-related psychological concerns in community-dwelling older people. Int Psychogeriatr. 2015;27:1071-87.

10. Hamel KA, Cavanagh PR. Stair performance in people aged 75 and older. $J$ Am Geriatr Soc. 2004;52:563-7.

11. Fortinsky RH, Panzer V, Wakefield D. Into F. Alignment between balance confidence and fall risk in later life: has over-confidence been overlooked? Health Risk Soc. 2009:11:341-52.

12. Butler AA, Lord SR, Taylor JL, Fitzpatrick RC. Ability versus hazard: risk-taking and falls in older people. J Gerontol A Biol Sci Med Sci. 2015;70:628-34.

13. Matchar DB, Duncan PW, Lien CT, Ong MEH, Lee M, Gao F, et al. Randomized controlled trial of screening, risk modification, and physical therapy to prevent falls among the elderly recently discharged from the emergency department to the community: the steps to avoid falls in the elderly study. Arch Phys Med Rehabil. 2017;98:1086-96.

14. Hill K, Schwarz J, Kalogeropoulos A, Gibson S. Fear of falling revisited. Arch Phys Med Rehabil. 1996:77:1025-9.

15. Clark RA, Bryant AL, Pua YH, McCrory P, Bennell K, Hunt M. Validity and reliability of the Nintendo Wii balance board for assessment of standing balance. Gait \& Posture. 2010;31:307-10.

16. Huurnink A, Fransz DP, Kingma I, van Dieen JH. Comparison of a laboratory grade force platform with a Nintendo Wii balance board on measurement of postural control in single-leg stance balance tasks. J Biomech. 2013:46:1392-5

17. Jeka J, Kiemel T, Creath R, Horak F, Peterka R. Controlling human upright posture: velocity information is more accurate than position or acceleration. J Neurophysiol. 2004;92:2368-79.

18. TH MC Jr, Castro VM, Cagan A, Roberson AM, Perlis RH. Validation of a risk stratification tool for fall-related injury in a state-wide cohort. BMJ Open. 2017;7:e012189.

19. Carpenter CR, Shelton E, Fowler S, Suffoletto B, Platts-Mills TF, Rothman RE, et al. Risk factors and screening instruments to predict adverse outcomes for undifferentiated older emergency department patients: a systematic review and meta-analysis. Acad Emerg Med. 2015;22:1-21.

20. Fabre $\mathrm{JM}$, Ellis $\mathrm{R}$, Kosma M, Wood RH. Falls risk factors and a compendium of falls risk screening instruments. J Geriatr Phys Ther. 2010;33:184-97.

21. Guralnik JM, Simonsick EM, Ferrucci L, Glynn RJ, Berkman LF, Blazer DG, et al. A short physical performance battery assessing lower extremity function: association with self-reported disability and prediction of mortality and nursing home admission. J Gerontol. 1994;49:M85-94.

22. Gomez F, Wu YY, Auais M, Vafaei A, Zunzunegui MV. A simple algorithm to predict falls in primary care patients aged 65 to 74 years: the international mobility in aging study. J Am Med Dir Assoc. 2017; doi:10.1016/j.jamda.2017. 03.021

23. Gomez JF, Curcio CL, Alvarado B, Zunzunegui MV, Guralnik J. Validity and reliability of the short physical performance battery (SPPB): a pilot study on mobility in the Colombian Andes. Colomb Med (Cali). 2013;44:165-71.

24. World Health Organization: Definition of a Fall. http://www.who.int/ mediacentre/factsheets/fs344/en/ (2016). Accessed 02 Sep 2016.

25. Mackenzie L, Byles J, D'Este C. Validation of self-reported fall events in intervention studies. Clin Rehabil. 2006:20:331-9.

26. Duncan PW, Sullivan KJ, Behrman AL, Azen SP, SS W, Nadeau SE, et al, Protocol for the locomotor experience applied post-stroke (LEAPS) trial: a randomized controlled trial. BMC Neurol. 2007;7:39.

27. Garcia PA, Dias JM, Silva SL, Dias RC. Prospective monitoring and selfreport of previous falls among older women at high risk of falls and fractures: a study of comparison and agreement. Braz J Phys Ther. 2015:19:218-26.

28. Harrell FE Jr. Regression modeling strategies: with applications to linear models, logistic and ordinal regression, and survival analysis. 3rd ed. New York: Springer; 2015.

29. Chang BH, Pocock S. Analyzing data with clumping at zero. An example demonstration. J Clin Epidemiol. 2000;53:1036-43.

30. Harrell Jr FE: Rms: Regression modeling strategies. R package version 4.4-1. Http://cran.R-project.Org/package=rms (2015). Accessed 02 Feb 2016.

31. Gettens S, Fulbrook P. Fear of falling: association between the modified falls efficacy scale, in-hospital falls and hospital length of stay. J Eval Clin Pract. 2015;21:43-50

32. Murray KJ, Hill K, Phillips B, Waterston JA. Pilot study of falls risk and vestibular dysfunction in older fallers presenting to hospital emergency departments. Disabil Rehabil. 2005;27:499-506.

33. O'Halloran AM, Penard N, Galli A, Fan CW, Robertson IH, Kenny RA. Falls and falls efficacy: the role of sustained attention in older adults. BMC Geriatr. 2011;11:85.

34. Delbaere K, Close JC, Brodaty H, Sachdev P, Lord SR. Determinants of disparities between perceived and physiological risk of falling among elderly people: cohort study. BMJ. 2010:341:C4165.

35. Rochat S, Bula CJ, Martin E, Seematter-Bagnoud L, Karmaniola A, Aminian K, et al. What is the relationship between fear of falling and gait in wellfunctioning older persons aged 65 to 70 years? Arch Phys Med Rehabil. 2010;91:879-84.

36. Filiatrault J, Belley A-M, Laforest S, Gauvin L, Richard L, Desrosiers J, et al. Fear of falling among seniors: a target to consider in occupational and physical therapy practice? Phys Occup Ther in Geriatr. 2013;31:197-213. 
37. Delbaere K, Sturnieks DL, Crombez G, Lord SR. Concern about falls elicits changes in gait parameters in conditions of postural threat in older people. J Gerontol A Biol Sci Med Sci. 2009;64:237-42.

38. Kwan MM, Tsang WW, Lin SI, Greenaway M, Close JC, Lord SR. Increased concern is protective for falls in Chinese older people: the chopstix fall risk study. J Gerontol A Biol Sci Med Sci. 2013;68:946-53.

39. Okubo Y, Seino S, Yabushita N, Osuka Y, Jung S, Nemoto M, et al. Longitudinal association between habitual walking and fall occurrences among community-dwelling older adults: analyzing the different risks of falling. Arch Gerontol Geriatr. 2015;60:45-51.

40. Quach L, Galica AM, Jones RN, Procter-Gray E, Manor B, Hannan MT, et al. The nonlinear relationship between gait speed and falls: the maintenance of balance, independent living, intellect, and zest in the elderly of Boston study. J Am Geriatr Soc. 2011;59:1069-73.

Submit your next manuscript to BioMed Central and we will help you at every step:

- We accept pre-submission inquiries

- Our selector tool helps you to find the most relevant journal

- We provide round the clock customer support

- Convenient online submission

- Thorough peer review

- Inclusion in PubMed and all major indexing services

- Maximum visibility for your research

Submit your manuscript at www.biomedcentral.com/submit
Biomed Central 